ESAIM: COCV 20 (2014) 612-631

DOI: $10.1051 / \mathrm{cocv} / 2013077$
ESAIM: Control, Optimisation and Calculus of Variations

www.esaim-cocv.org

\title{
ON INDECOMPOSABLE SETS WITH APPLICATIONS
}

\begin{abstract}
ANDREW LORENT ${ }^{1}$
Abstract. In this note we show the characteristic function of every indecomposable set $F$ in the plane is $B V$ equivalent to the characteristic function a closed set $\mathbb{F}$, i.e. $\left\|\mathbb{1}_{F}-\mathbb{1}_{\mathbb{F}}\right\|_{B V\left(\mathbb{R}^{2}\right)}=0$. We show by example this is false in dimension three and above. As a corollary to this result we show that for every $\epsilon>0$ a set of finite perimeter $S$ can be approximated by a closed subset $\mathbb{S}_{\epsilon}$ with finitely many indecomposable components and with the property that $H^{1}\left(\partial^{M} \mathbb{S}_{\epsilon} \backslash \partial^{M} S\right)=0$ and $\left\|\mathbb{1}_{S}-\mathbb{1}_{\mathbb{S}_{\epsilon}}\right\|_{B V\left(\mathbb{R}^{2}\right)}<\epsilon$. We apply this corollary to give a short proof that locally quasiminimizing sets in the plane are $B V_{l}$ extension domains.
\end{abstract}

Mathematics Subject Classification. 28A75.

Received May 14, 2013. Revised October 29, 2013.

Published online March 28, 2014.

\section{INTRODUCTION}

Sets of finite perimeter are the largest class of sets that permit a broad theory of analysis. They have wide application in the Calculus of Variations, PDE, image processing and fracture mechanics. In some sense the theory of sets of finite perimeter is an analogue for sets of what the theory of Sobolev functions is for functions. A very useful notion for Sobolev functions is the notion of precise representative for a function $f \in W^{1, p}$, this is a function $\tilde{f} \in W^{1, p}$ with $\|f-\tilde{f}\|_{W^{1, p}}=0$ and $\tilde{f}$ has additional smoothness and regularity properties. Another very useful result is Whitney's theorem that for any $\epsilon>0$ there exists a smooth function $\hat{f}$ such that $\|\hat{f}-f\|_{W^{1, p}}<\epsilon$. We prove analogues results for sets of finite perimeter in the plane and we apply them to give a short proof that quasiminimizing sets are $B V_{l}$ extension domains.

Recall a set $E$ is said to be of finite perimeter in domain $\Omega$ if it is measurable and

$$
\operatorname{Per}(E, \Omega):=\sup \left\{\int_{E} \operatorname{div} \phi: \phi \in\left[C_{c}^{1}(\Omega)\right]^{N},\|\phi\|_{\infty} \leq 1\right\}<\infty .
$$

A set is simply called a set of finite perimeter if it is of finite perimeter in $\mathbb{R}^{n}$ and we define $\operatorname{Per}(E):=$ $\operatorname{Per}\left(E, \mathbb{R}^{n}\right)$. When dealing with sets of finite perimeter it is common to work with the representative that has negligible points removed. Given a set $E$ we define $\operatorname{Neg}(E):=\left\{x \in E:\left|B_{r}(x) \cap E\right|=0\right.$ for some $\left.r>0\right\}$. Let $\widetilde{E}=E \backslash \operatorname{Neg}(E)$. By Proposition $3.3[1]$ we know $\left\|\mathbb{1}_{E}-\mathbb{1}_{\widetilde{E}}\right\|_{B V\left(\mathbb{R}^{2}\right)}=0$. If a set $F$ is such that $\operatorname{Neg}(F)=\emptyset$ we say $F$ is shaved.

\footnotetext{
Keywords and phrases. Sets of finite perimeter, indecomposable sets.

1 Mathematics Department, University of Cincinnati, 2600 Clifton Ave. Cincinnati OH 45221. lorentaw@uc. edu
} 
A set of finite perimeter is called indecomposable iff for any disjoint subsets $A, B \subset E$ such that $E=A \cup B$ we have $\operatorname{Per}(E)=\operatorname{Per}(A)+\operatorname{Per}(B)$ then either $|A|=0$ or $|B|=0$.

The main theorem we will establish in this note is the following.

Theorem 1.1. If $F \subset \mathbb{R}^{2}$ is a shaved indecomposable set then the closure $\bar{F}$ of $F$ has the property

$$
\left\|\mathbb{1}_{F}-\mathbb{1}_{\bar{F}}\right\|_{B V\left(\mathbb{R}^{2}\right)}=0 .
$$

Consequently the characteristic function of any indecomposable set is BV equivalent to the characteristic function of a closed set.

A straightforward corollary to this is:

Corollary 1.2. Suppose $E \subset \mathbb{R}^{2}$ is a set of finite perimeter, then for any $\epsilon>0$ we can find a closed subset $\mathbb{E} \subset E$ with finitely many indecomposable components such that $H^{1}\left(\partial^{M} \mathbb{E} \backslash \partial^{M} E\right)=0$ and

$$
\left\|\mathbb{1}_{E}-\mathbb{1}_{\mathbb{E}}\right\|_{B V\left(\mathbb{R}^{2}\right)}<\epsilon .
$$

We will show by example that Theorem 1.1 is false for indecomposable sets of finite perimeter in $\mathbb{R}^{n}$ for $n \geq 3$. Our example also shows that Theorem 7 of [2] for saturated indecomposable sets in the plane does not hold true in dimension three and above. It is unclear to us if Corollary 1.2 is true in dimension three and above.

Theorem 1.3. There exists a shaved set of finite perimeter $S \subset Q=(-1,1) \times(-1,1) \times(-1,1)$ with the following properties

(i) $S$ is connected and $S^{c}$ is connected. Hence $S$ is an indecomposable saturated set.

(ii) $|\bar{S} \backslash S|>\frac{3999}{4000}$.

(iii) $H^{2}\left(\partial^{M} S \backslash \phi\left(S^{2}\right)\right)>0$ for any Lipschitz map $\phi: S^{2} \rightarrow \mathbb{R}^{3}$.

Our main application of Theorem 1.1 will be to show that local quasiminimizers in the plane are $B V_{l}$ extension domains. This is already known as a consequence of the work of David-Semmes [5], however our proof is much shorter. Specifically we say a set $E$ of finite perimeter $E$ is a $K$-quasiminimal set in $\Omega$ iff for all open $U \subset \subset \Omega$ and all Borel sets $F, G \subset U$ we have $\operatorname{Per}(E, U) \leq K \operatorname{Per}((E \cup F) \backslash G, U)$. And we say a set $E$ of finite perimeter is locally $K$-quasiminimizing if there exists $\delta>0$ such that for any $x \in \partial E$ the set $E$ is a $K$-quasiminimal in $B_{\delta}(x)$. In the case where $E$ is bounded $\delta$ can be chosen depending on $x$.

Finally a set $E$ of finite perimeter is a $B V_{l}$ extension domain if and only if there are constants $c \geq 1$ and $\delta>0$ such that whenever $u \in B V(E)$ is such that the diameter of the support of $u$ is smaller than $\delta$, then there is a function $T u \in B V$ such that $\|D T u\| \leq c\|D u\|(E)$ and $T u=u$ on $E$.

Corollary 1.4. If $E \subset \mathbb{R}^{2}$ is a locally $K$-quasiminimizing set then it is a $B V_{l}$ extension domain.

Note also that if Corollary 1.2 were true in dimension three and above then the proof of Corollary 1.4 would work in these dimensions too. A generalization of Corollary 1.2 to higher dimensions could potentially be a useful technical tool in the study of sets of finite perimeter.

In addition we will obtain the following corollary which is also an easy corollary to Theorem 7 [2]. Firstly some definitions, Ambrosio et al. [2] (Def. 2) define a hole of a set of finite perimeter $S$ to be an indecomposable component of $S^{c}$ with finite measure. A set $S$ is called saturated (again see Def. 2, [2]) if it is the union of itself and all its holes.

Corollary 1.5 (To Thm. 1.1). Suppose $S \subset \mathbb{R}^{2}$ is a indecomposable saturated set then there exists an open set $\mathbb{S}$ such that $\left\|\mathbb{1}_{S}-\mathbb{1}_{\mathbb{S}}\right\|_{B V\left(\mathbb{R}^{2}\right)}=0$. 


\section{SKetCH OF PROOF OF MAIN THEOREM}

The proof of the Theorem 1.1 follows from three basic steps. Each follows from the last in a fairly natural way. We will firstly state the steps then sketch the reasons they hold afterwards.

Since $E$ is a shaved indecomposable set it has the property

$$
\left|B_{r}(x) \cap E\right|>0 \text { for any } x \in E, r>0 .
$$

Step 1. Let $Z:=\left\{z \in \mathbb{R}^{2}: \lim \sup _{r \rightarrow 0} \frac{\left|E \cap B_{r}(x)\right|}{\pi r^{2}} \geq \frac{1}{2}\right\}$. We will show we can find a countable collection of balls $\left\{B_{r_{n}}\left(x_{n}\right): x_{n} \in Z\right\}$ with the following properties.

(i) $\left\{B \frac{r_{n}}{5}\left(x_{n}\right): n \in \mathbb{N}\right\}$ are disjoint.

(ii) $Z \subset \cup_{n} B_{r_{n}}\left(x_{n}\right)$.

(iii) $\left|B_{r_{n}}\left(x_{n}\right) \cap E\right| \geq \frac{\pi r_{n}^{2}}{4}$ for each $n$.

(iv) $\Pi:=\bigcup_{n} B_{r_{n}}\left(x_{n}\right)$ is connected.

Step 2. We will show that for $H^{1}$ a.e. $x \in \partial E \backslash Z$ there exists $r_{x}>0$ such that

$$
H^{1}\left(\partial E \cap B_{r}(x)\right)>\frac{r}{1600} \text { for all } r \in\left(0, r_{x}\right] .
$$

Step 3. We will show that $H^{1}(\partial E \backslash Z)=0$.

Sketch of Step 1. By definition of $Z$ for any $z \in Z$ we can find $r_{z}>0$ such that $\left|B_{r}(z) \cap Z\right| \geq \frac{\pi r^{2}}{4}$ for all $r \in\left(0, r_{z}\right]$. So by the $5 r$ Covering Theorem we can find a subcollection $\left\{B_{r_{z_{n}}}\left(z_{n}\right): n \in \mathbb{N}\right\}$ such that $\left\{B \frac{r_{z_{n}}}{5}\left(z_{n}\right): n \in \mathbb{N}\right\}$ are pairwise disjoint and $Z \subset \bigcup_{n} B_{r_{z_{n}}}\left(z_{n}\right)$. The only remaining issue is to show that $\Pi:=\bigcup_{n} B_{r_{z_{n}}}\left(z_{n}\right)$ is connected. Suppose it is not, so there are two non-empty disjoint connect components of $\Pi_{0}$ and $\Pi_{1}$ such that $\Pi=\Pi_{0} \cup \Pi_{1}$. Letting $Q_{0}=E \cap \Pi_{0}$ and $Q_{1}=E \cap \Pi_{1}$ it is possible to show that $H^{1}\left(\partial^{M} Q_{0} \cap \partial^{M} Q_{1}\right)=0$ and this implies $E$ is not an indecomposable set, contradiction. Thus $\Pi$ is connected.

Sketch of Step 2. Now we can assume without loss of generality that $x \in E^{0}$. So we can find $p_{x}>0$ such that $\left|E \cap B_{r}(x)\right|<\frac{r^{2}}{10000}$ for all $r \in\left(0, p_{x}\right)$. Note also by (2.1) for any $x \in \partial E$ we have that $\left|E \cap B_{\frac{r}{1000}}(x)\right|>0$ for any $r>0$. So let $r \in\left(0, p_{x}\right)$, by Step 1 we have a countable collection of balls $\left\{B_{r_{n}}\left(x_{n}\right): n \in \mathbb{N}\right\}$ that satisfy (i), $(i i),(i i i),(i v)$. A subcollection of these balls $\left\{B_{r_{p_{k}}}\left(x_{p_{k}}\right): k \in \mathbb{N}\right\}$ is such that $B_{r_{p_{k}}}\left(x_{p_{k}}\right) \cap B_{r}(x) \neq \emptyset$ for any $k \in \mathbb{N}, E \cap B_{r}(x) \subset \bigcup_{k} B_{r_{p_{k}}}\left(x_{p_{k}}\right)$ and $\widetilde{\Pi}:=\bigcup_{k} B_{r_{p_{k}}}\left(x_{p_{k}}\right)$ is a connected set. So $\widetilde{\Pi}$ is a 'tentacle' of balls that reaches from the outside of $B_{r}(x)$ to $B_{\frac{r}{1000}}(x)$. And note that any ball $B_{r_{p_{k}}}\left(x_{p_{k}}\right)$ has at least a quarter of its area is filled by $E$. On the other hand most of $B_{r}(x)$ is empty of $E$. So assume for the moment for simplicity that the tentacle reaches into $B_{\frac{r}{100}}(x)$ in something like a line, pick a direction $v$ that is roughly orthogonal to the line. Now there must be a large set of lines in direction $v$ running through $B_{r}(x)$ that start at some point in $E \cap B_{r}(x)$ and end in some point in $E^{c} \cap B_{r}(x)$. The variation of $\mathbb{1}_{E}$ restricted to any of these lines is at least 1 , so integrating across these lines gives (2.2). Note the reason our proof works in $\mathbb{R}^{2}$ and does not work in higher dimension ${ }^{2}$ is that a 'tentacle' in higher dimension has arbitrarily small surface area, where as in two dimension the surface area of a tentacle is $O(1)$.

Sketch of Step 3. Suppose $H^{1}(\partial E \backslash Z)>0$. Assume for simplicity $H^{1}(\partial E \backslash Z)<\infty$. Let $\mu(A):=H^{1}\left(\partial^{M} E \cup\right.$ $(\partial E \backslash Z))$. We can find an open set $U$ with $\partial^{M} E \subset U$ and a compact set $C \subset \partial^{M} E$ such that $\mu(U) \leq \mu\left(\partial^{M} E\right)+\epsilon$ and $\mu(C) \geq \mu\left(\partial^{M} E\right)-\epsilon$. We can assume $\epsilon$ is sufficiently small so that $\mu(\partial E \backslash U)>\frac{H^{1}(\partial E \backslash Z)}{2}$.

\footnotetext{
${ }^{2}$ And recall indeed by the example constructed in Theorem 1.3 shows the result in false in higher dimension.
} 
Now since $\delta=\operatorname{dist}(\partial U, C)>0$ we can find a countable collection of pairwise disjoint balls $\left\{B_{r_{n}}\left(x_{n}\right): x_{n} \in \partial E \backslash U, r_{n}<\delta\right\}$ such that $\sum_{n} r_{n} \geq c_{0} \mu(\partial E \backslash U)$ some constant $c_{0}>0$. By Step 2 we have that $\sum_{n} H^{1}\left(\partial^{M} E \cap B_{r_{n}}\left(x_{n}\right)\right) \geq \frac{c_{0}}{1600} \mu(\partial E \backslash U)$. Since $B_{r_{n}}\left(x_{n}\right) \cap C=\emptyset$ for all $n$ we have

$$
\sum_{n} H^{1}\left(\partial^{M} E \cap B_{r_{n}}\left(x_{n}\right)\right)+H^{1}(C) \geq \frac{c_{0}}{1600} \mu(\partial E \backslash U)+H^{1}\left(\partial^{M} E\right)-\epsilon
$$

which is contradiction for small enough $\epsilon$.

\section{Sketch of Proof of the APplication to QUASIMINIMIZING SETS}

As stated the main application of Theorem 1.1 is Corollary 1.4. So to establish this we will use the criteria for $B V_{l}$ extension domains found in [3,4]. Namely $E$ is a $B V_{l}$ extension domain if for every set of finite perimeter $F \subset E$ with $\operatorname{diam}(F)<\gamma$ we can find $\hat{F}$ with $F \subset \hat{F}$ and $\operatorname{Per}\left(\hat{F}, \mathbb{R}^{2}\right) \leq C \operatorname{Per}(F, E)$. Following the method of (the preprint form of) [6] we take $\hat{F}$ to be equal to $F$ and it will suffice to show that

$$
\operatorname{Per}\left(\hat{F}, \mathbb{R}^{2}\right) \leq(1+K) \operatorname{Per}(F, E)
$$

for any set of finite perimeter $F \subset E$ with $\operatorname{diam}(F)<\gamma$. We will achieve this in the following way: for any $\delta>0$ we will find an open set $\Omega$ with $F \subset \subset \Omega$ and

$$
H^{1}\left(\partial^{M} E \cap \Omega\right) \leq H^{1}\left(\partial^{M} E \cap \partial^{M} F\right)+\delta .
$$

Then by the fact that $E$ is a local $K$-quasiminimizer $\operatorname{Per}(E, \Omega) \leq K \operatorname{Per}(E \backslash F, \Omega)$. Now

$$
\begin{aligned}
H^{1}\left(\partial^{M} E \cap \partial^{M} F\right) & \leq H^{1}\left(\partial^{M} E \cap \Omega\right) \\
& =\operatorname{Per}(E, \Omega) \\
& \leq K \operatorname{Per}(E \backslash F, \Omega) \\
& =K\left(H^{1}\left(\partial^{M} F \cap E\right)+H^{1}\left(\left(\partial^{M} E \backslash \partial^{M} F\right) \cap \Omega\right)\right) \\
& \stackrel{(3.2)}{\leq} K \operatorname{Per}(F, E)+K \delta .
\end{aligned}
$$

As this holds for arbitrary $\delta>0$ we have inequality (3.1). Note this inequality can not work unless we can show (3.2) and hence establish $H^{1}\left(\left(\partial^{M} E \backslash \partial^{M} F\right) \cap \Omega\right)<\delta$. Now for arbitrary sets of finite perimeter $F \subset E$ it is not true we can find $\Omega$ such that $F \subset \subset \Omega$ and (3.2) holds true. For a counter example let $E=B_{1}(0)$ and pick $z_{0} \in \partial E$, let $\alpha \in(0,1)$ and let $\zeta_{k}$ be the set of points with rational coordinates in $E \cap B_{\alpha}\left(z_{0}\right)$. Then the set $F=\bigcup_{k} B_{\alpha 2^{-(1000+n)}}\left(\zeta_{n}\right) \cap E$ is a set of finite perimeter for which (3.2) is false for any open set $\Omega$ with $F \subset \subset \Omega$.

However we will be able to carry out this argument by replacing $F$ by the set $\mathbb{F}$ afforded to us by Corollary 1.2 . The set $\mathbb{F}$ has almost the same characteristics of $F$ and we can find an open set $\Omega$ with $\mathbb{F} \subset \subset \Omega$ such that $H^{1}\left(\partial^{M} E \cap \Omega\right) \leq H^{1}\left(\partial^{M} E \cap \partial^{M} \mathbb{F}\right)+\delta$. So we can carry out the chain of inequalities to establish (3.3). Having established (3.3) for $\mathbb{F}$ the same inequality follows for $F$ with arbitrarily small error by (1.1). See Lemma 6.4 and Section 6.2 for full details.

\section{Preliminaries}

As in Definition $3.60[1]$ we every $t \in[0,1]$ we let $E^{t}$ denote the set of points of $t$ density, i.e.

$$
E^{t}:=\left\{x \in \mathbb{R}^{n}: \lim _{r \rightarrow 0} \frac{\left|E \cap B_{r}(x)\right|}{\Gamma(n) r^{n}}=t\right\}
$$

where $\Gamma(n)=\left|B_{1}(0)\right|$. 
It is a fundamental result of Federer (see Thm. 3.61 [1]) that if $E$ is a set of finite perimeter in $\Omega$, $H^{n-1}\left(\Omega \backslash\left(E^{0} \cup E^{\frac{1}{2}} \cup E^{1}\right)\right)=0$.

The measure theoretic boundary is defined by

$$
\partial^{M} E:=\left\{x \in \mathbb{R}^{n}: \limsup _{r \rightarrow 0} \frac{\left|E \cap B_{r}(x)\right|}{\left|B_{r}(x)\right|}>0 \text { and } \limsup _{r \rightarrow 0} \frac{\left|E^{c} \cap B_{r}(x)\right|}{\left|B_{r}(x)\right|}>0\right\} .
$$

It is well-known that $H^{n-1}\left(\partial^{M} E \backslash E^{\frac{1}{2}}\right)=0$ and $\left|D \mathbb{1}_{E}\right|=H_{\partial^{M} E}^{n-1}$, see Theorems 3.59 and 3.61 [1].

\section{Preliminary Lemmas}

Lemma 5.1. Suppose $E \subset \mathbb{R}^{2}$ is indecomposable. Then we can find a countable collection

$$
\left\{B_{r_{n}}\left(x_{n}\right): x_{n} \in E\right\}
$$

such that

$$
\begin{gathered}
\left(\bigcup_{n} B_{r_{n}}\left(x_{n}\right)\right) \text { is connected, } \\
\left|B_{r}\left(x_{n}\right) \cap E\right|>\frac{\pi r^{2}}{4} \text { for all } r \in\left(0, r_{n}\right], n \in \mathbb{N} .
\end{gathered}
$$

As a consequence for $H^{1}$ a.e. $x \in \partial E \backslash\left(E^{1} \cup \partial^{M} E\right)$ there exists $r_{x}>0$ such that

$$
H^{1}\left(\partial E \cap B_{r}(x)\right)>\frac{r}{1600} \text { for all } r \in\left(0, r_{x}\right] .
$$

Proof of Lemma 5.1. Let $Z=E^{1} \cup E^{\frac{1}{2}}$. By Theorem 3.61 [1]

$$
H^{1}(E \backslash Z)=0 .
$$

So for any $x \in Z$ there exists $r_{x}>0$ such that

$$
\left|B_{r}(x) \cap E\right|>\frac{\pi r^{2}}{4} \text { for all } r \in\left(0, r_{x}\right] .
$$

Step 1. By the $5 r$ Covering Theorem (see Thm. 2.11 [8]) we can find a disjoint sub-collection $\left\{B \frac{r_{x_{n}}}{5}\left(x_{n}\right): x_{n} \in Z\right\}$ such that $Z \subset \bigcup_{n} B_{r_{x_{n}}}\left(x_{n}\right)=: \Pi$. We will show $\Pi$ is connected.

Proof of Step 1. We argue by contradiction. Suppose $\Pi$ is disconnected. Let $\Pi_{0}$ be a connected non-empty component of $\Pi$ and let $\Pi_{1}=\Pi \backslash \Pi_{0}$. Now define $Q_{0}=E \cap \Pi_{0}$ and $Q_{1}=E \cap \Pi_{1}$. These are both the intersection of two sets of finite perimeter and hence are sets of finite perimeter.

We claim

$$
H^{1}\left(\left(E \backslash Q_{0}\right) \backslash Q_{1}\right)=0 .
$$

So let $\omega=E \backslash Z$, note $H^{1}(\omega) \stackrel{(5.4)}{=} 0$ and $E \backslash\left(Q_{0} \cup Q_{1}\right)=E \backslash \Pi \subset(Z \cup \omega) \backslash \Pi \subset \omega$. So equality (5.6) follows. We also claim

$$
Q_{1} \backslash\left(E \backslash Q_{0}\right)=\emptyset .
$$

Now $\left(Q_{1} \cup Q_{0}\right) \backslash E=\Pi \cap E \backslash E=\emptyset$ so (5.7) is immediate. So we actually have

$$
H^{1}\left(Q_{1} \triangle\left(E \backslash Q_{0}\right)\right)=0 .
$$


By Proposition 3.38 [1] this is more than enough to conclude

$$
\operatorname{Per}\left(Q_{1}\right)=\operatorname{Per}\left(E \backslash Q_{0}\right) .
$$

We will show

$$
H^{1}\left(Q_{0}^{\frac{1}{2}} \cap Q_{1}^{\frac{1}{2}}\right)=0
$$

Now if $x \in Q_{1}^{\frac{1}{2}}$ we must have

so

$$
\lim _{r \rightarrow 0} \frac{\left|E \cap \Pi_{1} \cap B_{r}(x)\right|}{\pi r^{2}}=\frac{1}{2}
$$

$$
\lim _{r \rightarrow 0} \frac{\left|E \cap B_{r}(x)\right|}{\pi r^{2}} \geq \frac{1}{2} .
$$

Hence (5.11) together with Theorem 3.61 [1] implies

so

$$
H^{1}\left(Q_{1}^{\frac{1}{2}} \backslash Z\right)=0
$$

$$
H^{1}\left(Q_{1}^{\frac{1}{2}} \backslash \Pi\right)=0 .
$$

Now note that if $x \in Q_{1}^{\frac{1}{2}} \cap \Pi$ we can not have $x \in \Pi_{0}$ because $\Pi_{0}$ is open and so $B_{\delta}(x) \subset \Pi_{0}$ for some $\delta>0$. By the fact $x \in Q_{1}^{\frac{1}{2}}$ also we must be able to find $y \in B_{\delta}(x) \cap Q_{1}$ which contradicts the fact $\Pi_{1}, \Pi_{0}$ are disjoint. Thus

$$
Q_{1}^{\frac{1}{2}} \cap \Pi \subset Q_{1}^{\frac{1}{2}} \cap \Pi_{1}
$$

And in the same way since $\Pi_{1}$ is open for all small enough $r$ we have that $B_{r}(x) \subset \Pi_{1}$. Thus if $x \in Q_{1}^{\frac{1}{2}} \cap \Pi_{1}$ by definition of $Q_{1}^{\frac{1}{2}}, \lim _{r \rightarrow 0} \frac{\left|\left(E \cap \Pi_{1}\right)^{c} \cap B_{r}(x)\right|}{\pi r^{2}}=\frac{1}{2}$ we actually have

$$
\lim _{r \rightarrow 0} \frac{\left|E^{c} \cap B_{r}(x)\right|}{\pi r^{2}}=\frac{1}{2}
$$

hence $x \in E^{\frac{1}{2}}$. Thus

$$
Q_{1}^{\frac{1}{2}} \cap \Pi_{1} \subset E^{\frac{1}{2}}
$$

Hence

$$
\begin{aligned}
H^{1} & \left(Q_{1}^{\frac{1}{2}} \backslash\left(E^{\frac{1}{2}} \cap \Pi_{1}\right)\right) \\
& \leq H^{1}\left(Q_{1}^{\frac{1}{2}} \backslash \Pi\right)+H^{1}\left(Q_{1}^{\frac{1}{2}} \cap \Pi \backslash Q_{1}^{\frac{1}{2}} \cap \Pi_{1}\right)+H^{1}\left(Q_{1}^{\frac{1}{2}} \cap \Pi_{1} \backslash E^{\frac{1}{2}} \cap \Pi_{1}\right) \\
& \quad \stackrel{(5.12),(5.13),(5.14)}{=} 0 .
\end{aligned}
$$

Now going in the opposite direction if $x \in E^{\frac{1}{2}} \cap \Pi_{1}$ again since $\Pi_{1}$ is open for all small enough $r$ we have that

$$
\lim _{r \rightarrow 0} \frac{\left|B_{r}(x) \cap E\right|}{\pi r^{2}}=\lim _{r \rightarrow 0} \frac{\left|B_{r}(x) \cap E \cap \Pi_{1}\right|}{\pi r^{2}}=\frac{1}{2}
$$

and

$$
\lim _{r \rightarrow 0} \frac{\left|B_{r}(x) \cap E^{c}\right|}{\pi r^{2}}=\lim _{r \rightarrow 0} \frac{\left|B_{r}(x) \cap\left(E \cap \Pi_{1}\right)^{c}\right|}{\pi r^{2}}=\frac{1}{2} .
$$

So $E^{\frac{1}{2}} \cap \Pi_{1} \subset\left(E \cap \Pi_{1}\right)^{\frac{1}{2}}=Q_{1}^{\frac{1}{2}}$ hence putting this together with (5.15) we have established

$$
H^{1}\left(E^{\frac{1}{2}} \cap \Pi_{1} \triangle Q_{1}^{\frac{1}{2}}\right)=0 .
$$


In exactly the same way we can show that $H^{1}\left(E^{\frac{1}{2}} \cap \Pi_{2} \triangle Q_{2}^{\frac{1}{2}}\right)=0$. Since $\Pi_{1}, \Pi_{0}$ are disjoint this completes the proof of (5.10).

Now $E=Q_{0} \cup Q_{1}$ and $\operatorname{Per}\left(Q_{0}\right)=H^{1}\left(Q_{0}^{\frac{1}{2}}\right), \operatorname{Per}\left(Q_{1}\right)=H^{1}\left(Q_{1}^{\frac{1}{2}}\right)$ and $\operatorname{Per}(E)=\operatorname{Per}\left(Q_{0}\right)+\operatorname{Per}\left(Q_{1}\right) \stackrel{(5.9)}{=}$ $\operatorname{Per}\left(Q_{0}\right)+\operatorname{Per}\left(E \backslash Q_{0}\right)$. So as $Q_{0}, E \backslash Q_{0}$ are both sets of finite perimeter this contradicts the fact $E$ is indecomposable. This concludes the proof of Step 1.

Step 2. We will establish (5.3).

Proof of Step 2. Firstly by Theorem 3.61 [1] we can assume $x \in E^{0}$. Now recall from the sketch of the proof (see property (2.1)), for any $y \in E$ we have $\left|B_{r}(y) \cap E\right|>0$ for any $r>0$. Thus since $x \in \partial E$ it has the same property. Now since $x \in E^{0}$ we can find $p_{x}>0$ such that

$$
\left|E \cap B_{r}(x)\right|<\frac{r^{2}}{100000} \text { for all } r \in\left(0, p_{x}\right) .
$$

However we must also have that $\left|E \cap B_{\frac{r}{1000}}(x)\right|>0$. So by property (i) we have established in Step 1 we can find a countable collection

$$
\left\{B_{r_{k}}\left(x_{k}\right): x_{k} \in E, r_{k}<\frac{r}{1000}\right\}
$$

such that $E^{1} \cup E^{\frac{1}{2}} \subset \bigcup_{k} B_{r_{k}}\left(x_{k}\right)$ and $\left(\bigcup_{k} B_{r_{k}}\left(x_{k}\right)\right)$ is connected. Now pick a point $x_{0} \in E^{1} \cap B_{\frac{r}{1000}}(x)$ and a point $y_{0} \in E^{1} \backslash B_{r}(x)$. Since $\bigcup_{k} B_{r_{k}}\left(x_{k}\right)$ is open and connected it is path connected and so we must be able to find a path $\phi:[0, t] \rightarrow \bigcup_{k} B_{r_{k}}\left(x_{k}\right)$ with $\phi(0)=x_{0}, \phi(t)=y_{0}$. Let $s \in(0, t)$ be the smallest number such that $\phi(s) \in \partial B_{\frac{r}{4}}(x)$, by compactness clearly this number exists. So

$$
\{\phi(w): 0 \leq w \leq s\} \subset B_{\frac{r}{4}}(x) .
$$

Let $v=\frac{\phi(s)-x_{0}}{\mid \phi(s)-x_{0}}$. For any vector $w$ let $\langle w\rangle:=\{\lambda w: \lambda \in \mathbb{R}\}$. Define $P_{V}(x)$ to be the orthogonal projection of $x$ onto subspace $V$. Note that for every $k$

$$
H^{1}\left(P_{\langle v\rangle}\left(B_{r}\left(x_{k}\right) \cap E\right)\right) \geq \frac{r}{16} \text { for all } r \in\left(0, r_{k}\right]
$$

since if this was not true we would have that $\left|B_{r}\left(x_{k}\right) \cap E\right| \leq \frac{r^{2}}{8}$ which contradicts (5.2).

We know $\phi([0, t]) \subset \bigcup_{k} B_{r_{k}}\left(x_{k}\right)$, let $\left\{B_{r_{p_{k}}}\left(x_{p_{k}}\right): k \in \mathbb{N}\right\}$ be a subcollection defined by

$$
\{\phi(w): 0 \leq w \leq s\} \cap B_{r_{p_{k}}}\left(x_{p_{k}}\right) \neq \emptyset \text { for any } k .
$$

So

$$
\{\phi(w): 0 \leq w \leq s\} \subset \bigcup_{k} B_{r_{p_{k}}}\left(x_{p_{k}}\right) .
$$

And

$$
\bigcup_{k} B_{r_{p_{k}}}\left(x_{p_{k}}\right) \stackrel{(5.19),(5.17)}{\subset} B_{\frac{r}{3}}(x)
$$

Now since

$$
P_{\langle v\rangle}(\{\phi(w): w \in[0, s]\}) \stackrel{(5.20)}{\subset} \bigcup_{k} P_{\langle v\rangle}\left(B_{r_{p_{k}}}\left(x_{p_{k}}\right)\right)
$$

by the $5 r$ Covering Theorem (see Thm. 2.11 [8]) we can find a subcollection

$$
\left\{B_{r_{q_{k}}}\left(x_{q_{k}}\right): k \in \mathbb{N}\right\}
$$


such that

$$
\left\{P_{\langle v\rangle}\left(B_{r_{p_{k}}}\left(x_{p_{k}}\right)\right): k \in \mathbb{N}\right\} \subset\left\{P_{\langle v\rangle}\left(B_{r_{q_{k}}}\left(x_{q_{k}}\right)\right): k \in \mathbb{N}\right\}
$$

and

$$
\left\{P_{\langle v\rangle}\left(B_{\frac{r_{q_{k}}}{5}}\left(x_{q_{k}}\right)\right): k \in \mathbb{N}\right\} \text { are disjoint. }
$$

Now as $H^{1}\left(P_{\langle v\rangle}(\{\phi(w): w \in[0, s]\})\right) \geq \frac{r}{5}$ so putting this together with (5.22) and (5.24) we have

$$
\begin{aligned}
\sum_{k} r_{q_{k}} & \stackrel{(5.24)}{=} \sum_{k} 2^{-1} H^{1}\left(P_{\langle v\rangle}\left(B_{r_{p_{k}}}\left(x_{p_{k}}\right)\right)\right) \\
& \stackrel{(5.22)}{\geq} 2^{-1} H^{1}\left(P_{\langle v\rangle}(\{\phi(w): w \in[0, s]\})\right) \\
& \geq \frac{r}{10} .
\end{aligned}
$$

So let $\mathcal{O}:=\bigcup_{k} B \frac{r_{q_{k}}}{5}\left(x_{q_{k}}\right) \cap E$. By (5.18), (5.24) and (5.25) we have

$$
\begin{aligned}
H^{1}\left(P_{\langle v\rangle}(\mathcal{O})\right) & \stackrel{(5.24)}{=} \sum_{k} H^{1}\left(P_{\langle v\rangle}\left(B \frac{r_{q_{k}}}{5}\left(x_{q_{k}}\right) \cap E\right)\right) \\
& \stackrel{(5.18)}{\geq} \sum_{k} \frac{r_{q_{k}}}{80} \\
& \stackrel{(5.25)}{\geq} \frac{r}{800} .
\end{aligned}
$$

Now by (5.21) $\mathcal{O} \subset B_{\frac{r}{3}}(x)$. We claim we can find a subset $\Pi \subset P_{\langle v\rangle}(\mathcal{O})$ with $|\Pi| \geq \frac{r}{1600}$ such that

$$
H^{1}\left(P_{\langle v\rangle}^{-1}(\omega) \cap E^{c} \cap A\left(x, \frac{r}{3}, r\right)\right)>0 \text { for any } \omega \in \Pi .
$$

Suppose this is not true. So there is a set $\Lambda \subset P_{\langle v\rangle}(\mathcal{O})$ where $|\Lambda| \geq \frac{r}{1600}$ such that $H^{1}\left(P_{\langle v\rangle}^{-1}(\omega) \cap E^{c} \cap A\left(0, \frac{r}{3}, r\right)\right)=0$ for all $\omega \in \Lambda$. Let $\Xi=\bigcup_{\omega \in \Lambda} P_{\langle v\rangle}^{-1}(\omega) \cap E \cap A\left(0, \frac{r}{3}, r\right)$ and by Fubini $|\Xi| \geq|\Lambda| \frac{2 r}{3} \geq \frac{r^{2}}{2400}$ and $\left|\Xi \cap E^{c}\right|=0$ and this contradicts (5.16). We have established (5.27).

Now by Theorem 3.103 [1] we know that

$$
\begin{aligned}
\frac{r}{1600} & \leq \int_{w \in \Pi} V\left(\mathbb{1}_{E}, P_{\langle v\rangle}^{-1}(w) \cap B_{r}(x)\right) \mathrm{d} w \\
& \leq V\left(\mathbb{1}_{E}, B_{r}(x)\right) \\
& =H^{1}\left(\partial^{M} E, B_{r}(x)\right) .
\end{aligned}
$$

So this establishes (5.3).

Proof of Theorem 1.1. Firstly as before, without loss of generality we can assume that for any $x \in F$, $\left|F \cap B_{\delta}(x)\right|>0$ for all $\delta>0$.

Step 1. We will show $H^{1}\left(\bar{F} \backslash\left(\partial^{M} F \cup F^{1}\right)\right)=0$.

Proof of Step 1. Suppose

$$
H^{1}\left(\bar{F} \backslash\left(\partial^{M} F \cup F^{1}\right)\right)>0 .
$$

Let $Z=\bar{F} \backslash\left(\partial^{M} F \cup F^{1}\right)$. Note that $Z \cap \operatorname{Int}(F)=\emptyset$ because if $x \in Z \cap \operatorname{Int}(F)$ then $x \in F^{1}$ which contradicts the definition of $Z$. So as $Z \subset \bar{F}$ we know that $Z \subset \partial F$ and thus $Z \subset \partial F \backslash \partial^{M} F$. 
Let $\epsilon>0$. If $H^{1}(Z)=\infty$ pick $B \subset Z$ with $H^{1}(B)=1$ and define $S=B \cup \partial^{M} F$ otherwise define $S=\left(\bar{F} \backslash F^{1}\right) \cup \partial^{M} F$. Let $\mu(A):=H^{1}(A \cap S)$. Note

$$
\mu\left(\partial F \backslash \partial^{M} F\right) \geq \min \left\{H^{1}\left(\bar{F} \backslash\left(F^{1} \cup \partial^{M} F\right)\right), H^{1}(B)\right\}=\beta>0 .
$$

Measure $\mu$ is Radon so we can find an open set $U$ such that $\partial^{M} F \subset U$ such that

$$
\mu(U)<\mu\left(\partial^{M} F\right)+\epsilon
$$

and we can find a compact set $C \subset \partial^{M} F$ such that

$$
\mu(C)>\mu\left(\partial^{M} F\right)-\epsilon
$$

And so

$$
\operatorname{dist}(C, \partial U)=\delta>0
$$

We can take $\varpi>0$ and a subset $\Gamma_{0} \subset \partial F \backslash U$ with

$$
H^{1}\left((\partial F \backslash U) \backslash \Gamma_{0}\right)<\epsilon
$$

and for any $r \in(0, \varpi), x \in \Gamma_{0}$ we have that $\mu\left(B_{r}(x)\right)=H^{1}\left(B_{r}(x) \cap S\right) \leq 2 r$. By Lemma 5.1 we can find $\sigma>0$, $\Gamma_{1} \subset \Gamma_{0}$ such that

$$
H^{1}\left(\partial^{M} F \cap B_{r}(x)\right)>\frac{r}{1600} \text { for all } x \in \Gamma_{1}, r \in(0, \sigma) .
$$

And

$$
H^{1}\left(\Gamma_{0} \backslash \Gamma_{1}\right)<\epsilon .
$$

Now by Vitali covering theorem (see Thm. 2.8 [8]) we can find a pairwise disjoint collection

$$
\left\{B_{r_{k}}\left(x_{k}\right): x_{k} \in \Gamma_{1}\right\}
$$

such that

$$
\mu\left(\Gamma_{1} \backslash\left(\bigcup_{k=1}^{\infty} B_{r_{k}}\left(x_{k}\right)\right)\right)=0
$$

and

$$
\sup \left\{r_{k}: k \in \mathbb{N}\right\}<\min \left\{\frac{\delta}{2}, \varpi, \sigma\right\}
$$

Note

$$
\begin{aligned}
\beta & \leq \mu\left(\partial F \backslash \partial^{M} F\right) \\
& =\mu(\partial F \backslash U)+\mu\left(\partial F \cap U \backslash \partial^{M} F\right) \\
& \leq \mu(\partial F \backslash U)+\mu\left(U \backslash \partial^{M} F\right) \\
& \quad \text { (5.30) } \\
& \leq \mu(\partial F \backslash U)+\epsilon .
\end{aligned}
$$

So $\mu(\partial F \backslash U)>\beta-\epsilon$. Now

$$
\sum_{k} \mu\left(B_{r_{k}}\left(x_{k}\right)\right) \stackrel{(5.36),(5.35),(5.33)}{\geq} \mu(\partial F \backslash U)-2 \epsilon \stackrel{(5.30)}{\geq} \mu\left(\partial F \backslash \partial^{M} F\right)-3 \epsilon .
$$

By (5.34) and the fact $x_{k} \in \Gamma_{1}, r_{k}<\sigma$ we have

$$
1600 H^{1}\left(\partial^{M} F \cap B_{r_{k}}\left(x_{k}\right)\right) \geq r_{k} \text { for all } k .
$$


Since we choose $r_{k}<\varpi$ and $x_{k} \in \Gamma_{1} \subset \Gamma_{0}$ be definition of $\Gamma_{0}, \mu\left(B_{r_{k}}\left(x_{k}\right)\right) \leq 2 r_{k}$ so

$$
\mu\left(B_{r_{k}}\left(x_{k}\right)\right) \leq 3200 H^{1}\left(\partial^{M} F \cap B_{r_{k}}\left(x_{k}\right)\right)
$$

thus putting this together with (5.38) we have

$$
\sum_{k} 3200 H^{1}\left(\partial^{M} F \cap B_{r_{k}}\left(x_{k}\right)\right) \geq \mu\left(\partial F \backslash \partial^{M} F\right)-3 \epsilon \stackrel{(5.29)}{\geq} \frac{\beta}{2} .
$$

Now since $x_{k} \in \Gamma_{1} \subset \partial F \backslash U$ by (5.32) and (5.37) we know that $C \cap B_{r_{k}}\left(x_{k}\right)=\emptyset$ for any $k$, so

$$
\begin{gathered}
H^{1}\left(\partial^{M} F\right) \geq H^{1}(C)+\sum_{k} H^{1}\left(B_{r_{k}}\left(x_{k}\right) \cap \partial^{M} F\right) \\
\stackrel{(5.40),(5.31)}{\geq} H^{1}\left(\partial^{M} F\right)+\frac{\beta}{6400}-\epsilon
\end{gathered}
$$

which is a contradiction assuming $\epsilon$ is small enough.

Step 2. We will show that $\bar{F}$ is a set of finite perimeter and $D \mathbb{1}_{\bar{F}}=D \mathbb{1}_{F}$.

Proof of Step 2. By Step $1\left|\bar{F} \backslash \partial^{M} F \cup F^{1}\right|=|\bar{F} \backslash F|=0$. So by Proposition 3.38 [1] we have $\operatorname{Per}(\bar{F})=\operatorname{Per}(F)$ and hence $\bar{F}$ is a set of finite perimeter and $D \mathbb{1}_{\bar{F}}=D \mathbb{1}_{F}$.

\section{The Applications}

\subsection{Quasiminimizing sets}

The following lemmas hold true in $\mathbb{R}^{n}$ without additional complexity, so we state them in $\mathbb{R}^{n}$.

Lemma 6.1. Given as set of finite perimeter $S$, suppose $H^{n-1}(A)=0$ then $\operatorname{Per}(S, A)=0$.

Proof Lemma 6.1. By Theorem 1.9 (2), Corollary 1.11 [8] measure $\mu$ defined by

$$
\mu(H):=H^{n-1}\left(\partial^{M} S \cap H\right)
$$

is a Radon measure.

Suppose set $A$ has the property $H^{n-1}(A)=0$. Then $\mu(A)=0$, so

$$
\begin{aligned}
0 & =\inf \{\mu(V): A \subset V, V \text { is open }\} \\
& =\inf \{\operatorname{Per}(S, V): A \subset V, V \text { is open }\} \\
& =\operatorname{Per}(S, A) .
\end{aligned}
$$

Using the fact $A \rightarrow \operatorname{Per}(S, A)$ is also Radon measure, see Proposition 3.38(a) and Proposition 1.43 [1].

Lemma 6.2. Let $E, F$ be sets of finite perimeter in $\mathbb{R}^{n}, F \subset E$. Then

$$
\operatorname{Per}\left(F, \partial^{M} E\right)=H^{n-1}\left(\partial^{M} F \cap \partial^{M} E\right) .
$$

and

$$
\operatorname{Per}\left(E, \partial^{M} F\right) \geq H^{n-1}\left(\partial^{M} F \cap \partial^{M} E\right) .
$$

Hence

$$
\operatorname{Per}\left(F, \partial^{M} E\right) \leq \operatorname{Per}\left(E, \partial^{M} F\right) .
$$


Proof of Lemma 6.2. Note that the measure $\nu(A):=H^{n-1}\left(\partial^{M} F \cap A\right)$ is a Radon measure. Letting $\sigma>0$ be some small number. Pick open $\Omega$ with

$$
\partial^{M} E \cap \partial^{M} F \subset \Omega
$$

such that

$$
\nu(\Omega)=H^{n-1}\left(\partial^{M} F \cap \Omega\right)<H^{n-1}\left(\partial^{M} E \cap \partial^{M} F\right)+\sigma=\nu\left(\partial^{M} E\right)+\sigma .
$$

Step 1. We will establish (6.1).

Proof of Step 1. Define

$$
B:=\left\{x \in\left(\partial^{M} F\right)^{c}: \limsup _{r \rightarrow 0} \frac{H^{n-1}\left(\partial^{M} F \cap B_{r}(x)\right)}{r^{n-1}}>0\right\}
$$

and

$$
D:=\left\{x \in \partial^{M} E: \liminf _{r \rightarrow 0} H^{n-1}\left(\partial^{M} E \cap B_{r}(x)\right) / r^{n-1}<1\right\} .
$$

By Theorem $6.2[8]$ we have that $H^{n-1}(B)=0$ and since $\partial^{M} E$ is rectifiable by Theorem $16.2[8] H^{n-1}(D)=0$.

So using Lemma 6.1 for the last equality

$$
\begin{aligned}
\operatorname{Per}\left(F, \partial^{M} E\right) & =\operatorname{Per}\left(F, \partial^{M} E \cap \partial^{M} F\right)+\operatorname{Per}\left(F, \partial^{M} E \backslash \partial^{M} F\right) \\
& =\operatorname{Per}\left(F, \partial^{M} E \cap \partial^{M} F\right)+\operatorname{Per}\left(F, \partial^{M} E \backslash\left(\partial^{M} F \cup B \cup D\right)\right) .
\end{aligned}
$$

Note $H^{n-1}\left(\partial^{M} E\right)<C$. Let $\epsilon>0$. We can find a decreasing sequence of number $\delta_{m} \rightarrow 0$ such that the sets

$$
\mathcal{U}_{m}:=\left\{\begin{aligned}
x \in \partial^{M} E \backslash\left(\partial^{M} F \cup B \cup D\right): & \frac{H^{n-1}\left(\partial^{M} F \cap B_{r}(x)\right)}{r^{n-1}}<\epsilon \\
& \text { and } \frac{H^{n-1}\left(\partial^{M} E \cap B_{r}(x)\right)}{r^{n-1}} \geq \frac{1}{2} \text { for all } r \in\left(0, \delta_{m}\right)
\end{aligned}\right\}
$$

have the property that $\partial^{M} E \backslash\left(\partial^{M} F \cup B \cup D\right) \subset \bigcup_{m=1}^{\infty} \mathcal{U}_{m}$.

Let $U_{1}=\mathcal{U}_{1} \backslash\left(\bigcup_{i=2}^{\infty} \mathcal{U}_{i}\right), U_{2}=\mathcal{U}_{2} \backslash\left(\bigcup_{i=3}^{\infty} \mathcal{U}_{i}\right), \ldots U_{k}=\mathcal{U}_{k} \backslash\left(\bigcup_{i=k+1}^{\infty} \mathcal{U}_{i}\right)$. Now $U_{l}, U_{k}$ are disjoint for any $l, k$ and

$$
\partial^{M} E \backslash\left(\partial^{M} F \cup B \cup D\right)=\bigcup_{i=1}^{\infty} \mathcal{U}_{i}=\bigcup_{i=1}^{\infty} U_{i} .
$$

Since $\left\{U_{1}, U_{2}, \ldots\right\}$ are pairwise disjoint

$$
\sum_{m} H^{n-1}\left(U_{m}\right) \stackrel{(6.9)}{<} H^{n-1}\left(\partial^{M} E \backslash \partial^{M} F\right) .
$$

Pick $m$. Since by Section 5.1 [8] we have $S^{1}\left(U_{m}\right) \leq 2 H^{1}\left(U_{m}\right)$ where $S^{1}$ denotes Spherical Hausdorff measure. So we can find a collection $\left\{B_{\frac{r_{k}}{2}}\left(z_{k}\right): r_{k}<\delta_{m}\right\}$ such that $U_{m} \subset \bigcup_{k} B_{\frac{r_{k}}{2}}\left(z_{k}\right)$ and $\sum_{k} \Gamma(n-1)\left(\frac{r_{k}}{2}\right)^{n-1} \leq$ $2 S^{1}\left(U_{m}\right)$. Now for each $k$ we can pick $x_{k} \in B_{\frac{r_{k}}{2}}\left(z_{k}\right) \cap U_{m}$ and then we have a collection of balls

$$
\left\{B_{r_{k}}\left(x_{k}\right): x_{k} \in U_{m}, r_{k}<\delta_{m}\right\}
$$

such that

$$
U_{m} \subset \bigcup_{k} B_{r_{k}}\left(x_{k}\right)=: V_{m}
$$

and

$$
\sum_{k} r_{k}^{n-1} \leq c S^{1}\left(U_{m}\right) \leq c H^{1}\left(U_{m}\right)
$$


Hence using the fact that $x_{k} \in U_{m} \subset \mathcal{U}_{m}$ we have

$$
\begin{aligned}
H^{n-1}\left(V_{m} \cap \partial^{M} F\right) & \stackrel{(6.12)}{\leq} \sum_{k} H^{n-1}\left(\partial^{M} F \cap B_{r_{k}}\left(x_{k}\right)\right) \\
\stackrel{(6.11),(6.8)}{\leq} & c \sum_{k} \epsilon r_{k}^{n-1} \\
& \stackrel{(6.13)}{\leq} c \in H^{n-1}\left(U_{m}\right) .
\end{aligned}
$$

Now

$$
\begin{aligned}
\operatorname{Per}\left(F, \partial^{M} E \backslash\left(\partial^{M} F \cup B \cup D\right)\right) & \stackrel{(6.9),(6.12)}{\leq} \operatorname{Per}\left(F, \bigcup_{m} V_{m}\right) \\
& \leq \sum_{m} H^{n-1}\left(V_{m} \cap \partial^{M} F\right) \\
& \stackrel{(6.14)}{\leq} \sum_{m} c \epsilon H^{n-1}\left(U_{m}\right) \\
& \stackrel{(6.10)}{\leq} c \in H^{n-1}\left(\partial^{M} E \backslash \partial^{M} F\right) .
\end{aligned}
$$

Taking the limit as $\epsilon \rightarrow 0$ we have $\operatorname{Per}\left(F, \partial^{M} E \backslash\left(\partial^{M} F \cup B \cup D\right)\right)=0$. So putting this together (6.7) we have

$$
\operatorname{Per}\left(F, \partial^{M} E\right)=\operatorname{Per}\left(F, \partial^{M} E \cap \partial^{M} F\right)
$$

And

$$
\begin{aligned}
\operatorname{Per}\left(F, \partial^{M} E \cap \partial^{M} F\right) & \stackrel{(6.4)}{\leq} \operatorname{Per}(F, \Omega) \\
& \leq H^{n-1}\left(\partial^{M} F \cap \Omega\right) \\
& \stackrel{(6.5)}{\leq} H^{n-1}\left(\partial^{M} F \cap \partial^{M} E\right)+\sigma
\end{aligned}
$$

Now as $\sigma$ is arbitrary, from (6.17) and (6.16), $\operatorname{Per}\left(F, \partial^{M} E\right) \leq H^{n-1}\left(\partial^{M} F \cap \partial^{M} E\right)$. Conversely for any open set $\Omega$ with $\partial^{M} E \subset \Omega$ we have $\operatorname{Per}(F, \Omega) \geq H^{n-1}\left(\partial^{M} F \cap \partial^{M} E\right)$. Thus by taking the infimum over all such open sets we have $\operatorname{Per}\left(F, \partial^{M} E\right) \geq H^{n-1}\left(\partial^{M} F \cap \partial^{M} E\right)$ and this completes the proof of Step 1 .

Step 2. We will establish (6.2).

Proof of Step 2. First note that $\partial^{M} E \cap \partial^{M} F$ is an $(n-1)$ rectifiable set. Let $\delta>0$, for $H^{n-1}$ a.e. $x \in \partial^{M} E \cap \partial^{M} F$ there exists $r_{x}>0$ such that

$$
\left|H^{n-1}\left(\partial^{M} F \cap \partial^{M} E \cap B_{h}(x)\right)-n \Gamma(n) h^{n-1}\right|<\delta h^{n-1} \text { for any } h \in\left(0, r_{x}\right) .
$$

Let $\alpha>0$. Defining $\Gamma_{0}:=\left\{x \in \partial^{M} F \cap \partial^{M} E: \alpha<r_{x}\right\}$ for all small enough $\alpha>0$ we have that

$$
H^{n-1}\left(\partial^{M} F \cap \partial^{M} E \backslash \Gamma_{0}\right)<\delta
$$

Since $A \rightarrow \operatorname{Per}(E, A)$ is a Radon measure we can extract a compact subset $\Gamma_{1} \subset \Gamma_{0}$ such that

$$
\operatorname{Per}\left(E, \Gamma_{1}\right)+\delta>\operatorname{Per}\left(E, \Gamma_{0}\right)
$$


And we can find a compact subset $\Gamma_{2} \subset \Gamma_{0}$ such that $H^{n-1}\left(\Gamma_{2}\right)+\delta>H^{n-1}\left(\Gamma_{0}\right)$. Let $\Gamma_{3}=\Gamma_{1} \cup \Gamma_{2}$. So $\Gamma_{3}$ is compact and has the properties

$$
\operatorname{Per}\left(E, \Gamma_{3}\right)+\delta>\operatorname{Per}\left(E, \Gamma_{0}\right) \text { and } H^{n-1}\left(\Gamma_{3}\right)+\delta>H^{n-1}\left(\Gamma_{0}\right) .
$$

And

$$
\Gamma_{3} \subset \Gamma_{0} \subset \partial^{M} F \cap \partial^{M} E .
$$

Again since we are dealing with a Radon measure we can find an open set $U$ with $\Gamma_{3} \subset U$ and

$$
\operatorname{Per}(E, U)<\operatorname{Per}\left(E, \Gamma_{3}\right)+\delta .
$$

Now $\operatorname{dist}\left(\Gamma_{3}, U\right)=: \beta>0$ by Vitali covering theorem we can find a collection of pairwise disjoint balls $\left\{B_{r_{k}}\left(x_{k}\right): x_{k} \in \Gamma_{3}, k \in \mathbb{N}\right\}$ where

$$
\sup \left\{r_{k}: k \in \mathbb{N}\right\}<\min \{\beta, \alpha\}
$$

and

$$
H^{n-1}\left(\Gamma_{3} \backslash\left(\bigcup_{k=1}^{\infty} B_{r_{k}}\left(x_{k}\right)\right)\right)=0 .
$$

Now since $\bigcup_{k=1}^{\infty} B_{r_{k}}\left(x_{k}\right) \subset U$ we know

$$
\operatorname{Per}\left(E, \bigcup_{k=1}^{\infty} B_{r_{k}}\left(x_{k}\right)\right) \leq \operatorname{Per}(E, U) \stackrel{(6.22)}{<} \operatorname{Per}\left(E, \Gamma_{3}\right)+\delta .
$$

But as $\Gamma_{3} \subset \Gamma_{0}$

$$
\begin{aligned}
\operatorname{Per}\left(E, \bigcup_{k=1}^{\infty} B_{r_{k}}\left(x_{k}\right)\right) & =\sum_{k=1}^{\infty} \operatorname{Per}\left(E, B_{r_{k}}\left(x_{k}\right)\right) \\
& =\sum_{k=1}^{\infty} H^{n-1}\left(\partial^{M} E \cap B_{r_{k}}\left(x_{k}\right)\right) \\
& \stackrel{(6.18)}{\geq} \sum_{k=1}^{\infty}(n \Gamma(n)-\delta) r_{k}^{n-1} .
\end{aligned}
$$

Now recall $\Gamma_{3} \subset \Gamma_{0} \subset \partial^{M} E \cap \partial^{M} F$ so

$$
\begin{aligned}
\sum_{k=1}^{\infty} n \Gamma(n)(1+\delta) r_{k}^{n-1} & \stackrel{(6.18)}{\geq} \sum_{k=1}^{\infty} H^{n-1}\left(\partial^{M} E \cap \partial^{M} F \cap B_{r_{k}}\left(x_{k}\right)\right) \\
& \stackrel{(6.21)}{\geq} \sum_{k=1}^{\infty} H^{n-1}\left(\Gamma_{3} \cap B_{r_{k}}\left(x_{k}\right)\right) \\
& \stackrel{(6.23)}{=} H^{n-1}\left(\Gamma_{3}\right) \\
& (6.20) \\
& \geq H^{n-1}\left(\Gamma_{0}\right)-\delta \\
& \stackrel{(6.19)}{\geq} H^{n-1}\left(\partial^{M} E \cap \partial^{M} F\right)-2 \delta
\end{aligned}
$$

Now putting (6.24)-(6.26) together we have

$$
\operatorname{Per}\left(E, \Gamma_{3}\right) \geq H^{n-1}\left(\partial^{M} E \cap \partial^{M} F\right)-3 \delta-c \sum_{k=1}^{\infty} \delta r_{k}^{n-1} .
$$


Now since the collection of balls $\left\{B_{r_{k}}\left(x_{k}\right): x_{k} \in \Gamma_{3}, k \in \mathbb{N}\right\}$ are pairwise disjoint and $x_{k} \in \Gamma_{3}$ we know by $(6.18)$ we know $\operatorname{Per}\left(E, B_{r_{k}}\left(x_{k}\right)\right) \geq \frac{n \Gamma(n) r_{k}^{n-1}}{2}$

$$
\begin{aligned}
\frac{n \Gamma(n)}{2} \sum_{k} r_{k}^{n-1} & \leq \sum_{k} \operatorname{Per}\left(E, B_{r_{k}}\left(x_{k}\right)\right) \\
& \leq \operatorname{Per}\left(E, \mathbb{R}^{n}\right) .
\end{aligned}
$$

So

$$
\sum_{k} r_{k}^{n-1} \leq c \operatorname{Per}\left(E, \mathbb{R}^{n}\right)
$$

Now putting (6.27) and (6.28) together we have

$$
\operatorname{Per}\left(E, \Gamma_{3}\right) \geq H^{n-1}\left(\partial^{M} E \cap \partial^{M} F\right)-3 \delta-c \delta \operatorname{Per}\left(E, \mathbb{R}^{n}\right) .
$$

Since Per $\left(E, \partial^{M} F\right) \geq \operatorname{Per}\left(E, \Gamma_{3}\right)$ and $\delta$ is arbitrarily small this establishes (6.2).

Proof of Lemma completed. By applying Step 1 and Step 2 we have

$$
\begin{aligned}
\operatorname{Per}\left(E, \partial^{M} F\right) & \stackrel{(6.2)}{\geq} H^{n-1}\left(\partial^{M} E \cap \partial^{M} F\right) \\
& \stackrel{(6.1)}{=} \operatorname{Per}\left(F, \partial^{M} E\right)
\end{aligned}
$$

Lemma 6.3. We will show that if $E \subset \mathbb{R}^{n}$ is an open set of finite perimeter and $\mathbb{F} \subset E$ is a relatively closed set of finite perimeter, then

$$
\partial^{M}(E \backslash \mathbb{F})=\left(\partial^{M} \mathbb{F} \cap E\right) \cup\left(\partial^{M} E \backslash \partial^{M} \mathbb{F}\right) .
$$

Proof. The lemma above holds true for arbitrary sets of finite perimeter $E$ and $\mathbb{F} \subset E$, we will only need it for open set $E$ and relatively closed set $\mathbb{F}$ and as the proof is easier in this case we argue only this result.

Let

$$
\Gamma_{1}=\partial^{M}(E \backslash \mathbb{F}) \cap \mathbb{F} \text { and } \Gamma_{2}=\partial^{M}(E \backslash \mathbb{F}) \backslash \mathbb{F} .
$$

Since $\mathbb{F}$ is relatively closed, if $x \in \Gamma_{2}$ then for some small enough $\delta>0,\left(B_{\delta}(x) \cap E\right) \cap \mathbb{F}=\emptyset$ so

$$
\lim _{r \rightarrow 0} \frac{\left|B_{r}(x) \cap(E \backslash \mathbb{F})\right|}{\Gamma(n) r^{n}}=\lim _{r \rightarrow 0} \frac{\left|B_{r}(x) \cap E\right|}{\Gamma(n) r^{n}}=\frac{1}{2}
$$

and thus $\Gamma_{2} \subset \partial^{M} E$ and hence $\Gamma_{2} \subset\left(\partial^{M} E \backslash \mathbb{F}\right) \subset \partial^{M} E \backslash \partial^{M} \mathbb{F}$. On the other hand $\Gamma_{1} \subset \mathbb{F} \subset E$ and $E$ is open. By definition of $\partial^{M}(E \backslash \mathbb{F})$ for any $x \in \Gamma_{1}$, for all small enough $r$ we have $\left|B_{r}(x) \cap(E \backslash \mathbb{F})\right| \approx \frac{\Gamma(n) r^{n}}{2}$ and $\left|B_{r}(x) \cap(E \backslash \mathbb{F})^{c}\right| \approx \frac{\Gamma(n) r^{n}}{2}$. But as $E$ is open $B_{r}(x) \subset E$ for all small enough $r$ so we must have $x \in \partial^{M} \mathbb{F}$. Thus $\Gamma_{1} \subset \partial^{M} \mathbb{F} \cap E$ and hence as $\Gamma_{1} \cup \Gamma_{2}=\partial^{M}(E \backslash \mathbb{F})$ this establishes (6.31).

Lemma 6.4. Let $E \subset \mathbb{R}^{n}$ be a locally $K$-quasiminimizing set and let $\mathbb{F} \subset E$ be a relatively closed subset of finite perimeter. Then

$$
\operatorname{Per}\left(\mathbb{F}, \partial^{M} E\right) \leq K \operatorname{Per}(\mathbb{F}, E) .
$$

Proof of Lemma 6.4. First note by Theorem $4.2[6]$ we know the topological boundary $\partial E$ is equal to the measure theoretic boundary $\partial^{M} E$.

Step 1. We will show there exists open set $\Omega$ with $\mathbb{F} \subset \Omega$ such that

$$
H^{n-1}\left(\left(\partial E \backslash \partial^{M} \mathbb{F}\right) \cap \Omega\right) \leq \epsilon .
$$


Proof of Step 1. Let $\mu(A):=H^{n-1}(A \cap \partial E)$. So $\mu$ is a Radon measure on $\mathbb{R}^{n}$ and hence we must be able to find open set $U$ with $\partial E \cap \partial \mathbb{F} \subset U$ such that $\mu(U) \leq H^{n-1}(\partial E \cap \partial \mathbb{F})+\epsilon$.

Note $\partial \mathbb{F} \cap \partial E$ is a closed set. So $\delta=\inf \{|x-y|: x \in \partial \mathbb{F} \cap \partial E, y \notin U\}>0$. Let $\Pi=\bigcup_{x \in \partial \mathbb{F} \cap \partial E} B_{\delta}(x)$. Note

$$
\mu(\Pi) \leq \mu(U) \leq H^{n-1}\left(\partial E \cap \partial^{M} F\right)+\epsilon .
$$

Note by compactness there exists $\lambda>0$ such that $N_{\lambda}(\partial E) \cap \mathbb{F} \subset \Pi$. Let $\Lambda=\bigcup_{x \in \mathbb{F} \backslash N_{\lambda}(\partial E)} B_{\frac{\lambda}{2}}(x)$. Note $\Lambda \cap \partial E=\emptyset$. And

$$
\mathbb{F}=\left(\mathbb{F} \cap N_{\lambda}(\partial E)\right) \cup\left(\mathbb{F} \backslash N_{\lambda}(\partial E)\right) \subset \Pi \cup \Lambda .
$$

Now

$$
\begin{gathered}
\mu(\Lambda \cup \Pi) \underset{(6.35)}{\leq} H^{n-1}\left(\partial E \cap \partial^{M} \mathbb{F}\right)+\epsilon .
\end{gathered}
$$

So letting $\Omega=\Lambda \cup \Pi$, open set $\Omega$ satisfies (6.34).

Proof of Lemma completed. Note that for any open set $\mathcal{O}$ with $E \subset \mathcal{O}$,

$$
H^{n-1}\left(\partial^{M} \mathbb{F} \cap E \cap \Omega\right) \leq H^{n-1}\left(\partial^{M} \mathbb{F} \cap E\right) \leq \operatorname{Per}(\mathbb{F}, \mathcal{O}) .
$$

Thus

$$
\operatorname{Per}(\mathbb{F}, E) \geq H^{n-1}\left(\partial^{M} \mathbb{F} \cap E \cap \Omega\right) .
$$

So using Lemma 6.2

$$
\begin{gathered}
\operatorname{Per}(E \backslash \mathbb{F}, \Omega) \stackrel{\stackrel{(6.31)}{=}}{\stackrel{(6.36),(6.34)}{\leq}} H^{1}\left(\partial^{M} \mathbb{F} \cap E \cap \Omega\right)+H^{1}\left(\left(\partial^{M} E \backslash \partial^{M} \mathbb{F}\right) \cap \Omega\right) \\
\operatorname{Per}(\mathbb{F}, E)+\epsilon .
\end{gathered}
$$

Now since $\mathbb{F} \subset \subset \Omega$ and $E$ is a quasiminimizer

$$
\begin{aligned}
\operatorname{Per}(E, \Omega) & \leq K \operatorname{Per}(E \backslash \mathbb{F}, \Omega) \\
& \stackrel{(6.37)}{=} K(\operatorname{Per}(\mathbb{F}, E)+\epsilon)
\end{aligned}
$$

And since $\mathbb{F}$ is closed, $\partial^{M} \mathbb{F} \subset \mathbb{F}$ so

$$
\operatorname{Per}\left(\mathbb{F}, \partial^{M} E\right) \stackrel{(6.3)}{\leq} \operatorname{Per}\left(E, \partial^{M} \mathbb{F}\right) \leq \operatorname{Per}(E, \mathbb{F}),
$$

thus

$$
\begin{aligned}
\operatorname{Per}\left(\mathbb{F}, \partial^{M} E\right)+\operatorname{Per}(E, \Omega \backslash \mathbb{F}) & \stackrel{(6.39)}{\leq} \operatorname{Per}(E, \mathbb{F})+\operatorname{Per}(E, \Omega \backslash \mathbb{F}) \\
& =\operatorname{Per}(E, \Omega) \\
& \stackrel{(6.38)}{\leq} K \operatorname{Per}(\mathbb{F}, E)+K \epsilon .
\end{aligned}
$$

Since this gives

$$
\operatorname{Per}\left(\mathbb{F}, \partial^{M} E\right) \leq K \operatorname{Per}(\mathbb{F}, E)+K \epsilon .
$$

And as $\epsilon$ is arbitrary this establishes (6.33). 


\subsection{Proof of Theorem 1.4.}

As stated in Section 3 by the criteria for $B V_{l}$ extension domain of [3,4], $E$ is a $B V_{l}$ extension domain if for every set of finite perimeter $F \subset E$ with $\operatorname{diam}(F)<\delta$ we can find $\hat{F}$ with $F \subset \hat{F}$ with $\operatorname{Per}\left(\hat{F}, \mathbb{R}^{n}\right) \leq(1+K) \operatorname{Per}(F, E)$. We will take $\hat{F}=F$ and we will show

$$
\operatorname{Per}\left(F, \mathbb{R}^{2}\right) \leq(1+K) \operatorname{Per}(F, E) .
$$

So start using Corollary 1.2 we can take closed subset $\mathbb{F} \subset F$ with $H^{1}\left(\partial^{M} \mathbb{F} \backslash \partial^{M} F\right)=0$ and

$$
\left\|\mathbb{1}_{\mathbb{F}}-\mathbb{1}_{F}\right\|_{B V}<\epsilon
$$

Now by Lemma 6.4

$$
\operatorname{Per}\left(\mathbb{F}, \partial^{M} E\right) \leq K \operatorname{Per}(\mathbb{F}, E) .
$$

Note since $\mathbb{F}$ is closed, $\operatorname{Per}\left(\mathbb{F}, \mathbb{F}^{c}\right)=0$. And note since $\mathbb{F} \subset E$ we know $H^{1}\left(E^{0} \cap \mathbb{F}\right)=0$ and so $\operatorname{Per}\left(\mathbb{F}, E^{0} \cap \mathbb{F}\right)=0$ by Lemma 6.1 . So

$$
\operatorname{Per}\left(\mathbb{F}, E^{0}\right)=\operatorname{Per}\left(\mathbb{F}, E^{0} \cap \mathbb{F}^{c}\right)+\operatorname{Per}\left(\mathbb{F}, E^{0} \cap \mathbb{F}\right)=0 .
$$

Now again by Lemma 6.1

$$
\operatorname{Per}\left(\mathbb{F}, \mathbb{R}^{2}\right) \leq \operatorname{Per}\left(\mathbb{F}, E^{1}\right)+\operatorname{Per}\left(\mathbb{F}, \partial^{M} E\right)+\operatorname{Per}\left(\mathbb{F}, E^{0}\right) \stackrel{(6.43),(6.44)}{\leq}(1+K) \operatorname{Per}(\mathbb{F}, E) .
$$

Hence

$$
\operatorname{Per}\left(F, \mathbb{R}^{2}\right) \stackrel{(6.42)}{\leq} \operatorname{Per}\left(\mathbb{F}, \mathbb{R}^{2}\right)+\epsilon \stackrel{(6.45)}{\leq}(1+K) \operatorname{Per}(\mathbb{F}, E)+\epsilon .
$$

For any open set $\mathcal{O}$ with $E \subset \mathcal{O}$ we have

$$
\operatorname{Per}(\mathbb{F}, E) \leq \operatorname{Per}(\mathbb{F}, \mathcal{O})=H^{1}\left(\partial^{M} \mathbb{F}, \mathcal{O}\right) \leq H^{1}\left(\partial^{M} F, \mathcal{O}\right)=\operatorname{Per}(F, \mathcal{O}) .
$$

As this is true for all open sets $\mathcal{O}$ with $E \subset \mathcal{O}$ we have $\operatorname{Per}(\mathbb{F}, E) \leq \operatorname{Per}(F, E)$ thus putting this into (6.46) we have $\operatorname{Per}\left(F, \mathbb{R}^{2}\right) \leq(1+K) \operatorname{Per}(F, E)+\epsilon$ and this holds for all $\epsilon>0$, so this establishes (6.41).

\subsection{Saturated indecomposable sets.}

Proof of Corollary 1.5.

Step 1. First we show that $|S|<\infty$.

Proof of Step 1. By the isoperimetric inequality (see [1] Thm. 46) we have that

$$
\min \left\{|S|,\left|\mathbb{R}^{2} \backslash S\right|\right\} \leq(4 \pi)^{-1}\left(\operatorname{Per}\left(S, \mathbb{R}^{2}\right)\right)^{2}
$$

Now if $\left|\mathbb{R}^{2} \backslash S\right| \leq(4 \pi)^{-1}\left(\operatorname{Per}\left(S, \mathbb{R}^{2}\right)\right)^{2}$ then $\mathbb{R}^{2} \backslash S$ must have an indecomposable component with finite measure which contradicts the definition of saturated, so this can not happen.

Step 2. Let

$$
a=\inf \left\{\tau>0:\left|D \mathbb{1}_{S}\right|\left(B_{\tau}(0)\right)>0\right\} \text { and } b=\sup \left\{\omega>0:\left|D \mathbb{1}_{S}\right|\left(\mathbb{R}^{2} \backslash B_{\omega}(0)\right)>0\right\} .
$$

We will show

$$
H^{1}\left(\partial^{M} S \cap \partial B_{s}\right)>0 \text { or } H^{1}\left(S \cap \partial B_{s}\right)>0 \text { for all } s \in(a, b) .
$$


Proof of Step 2. Suppose not, so for some $s \in(a, b)$ we have that

$$
H^{1}\left(\partial^{M} S \cap \partial B_{s}\right)=0 \text { and } H^{1}\left(S \cap \partial B_{s}\right)=0 .
$$

Now by definition of $a, b$ we know $\operatorname{Per}\left(S, B_{s}\right)>0$ and $\operatorname{Per}\left(S, \bar{B}_{s}{ }^{c}\right)>0$. Now let $A=S \cap B_{s}$ and $B=S \cap \bar{B}_{s}{ }^{c}$. So $A, B$ are both sets of finite perimeter.

Now for $H^{1}$ a.e. $x \in \partial^{M} A \backslash \partial^{M} S$, since $A \subset S$ we must have $x \in S^{1}$ (recall definition (4.1)) and so $x \in \partial B_{s}$. Thus

$$
H^{1}\left(\partial^{M} A \backslash\left(\partial^{M} S \cap \bar{B}_{s} \cup\left(S \cap \partial B_{s}\right)\right)\right)=0 .
$$

In the same way

$$
H^{1}\left(\partial^{M} B \backslash\left(\partial^{M} S \cap B_{s}^{c} \cup\left(S \cap \partial B_{s}\right)\right)\right)=0 .
$$

Thus by (6.48) we have that $H^{1}\left(\partial^{M} A \cap \partial^{M} B\right)=0$ and as $|A \cap B|=0$ by Proposition 1 [2] we have $\operatorname{Per}(A)+$ $\operatorname{Per}(B)=\operatorname{Per}(S)$ which contradicts the fact that $S$ is indecomposable.

Now suppose $b=\infty$, then letting

$$
H_{1}:=\left\{s>a: H^{1}\left(\partial^{M} S \cap \partial B_{s}\right)>0\right\} .
$$

and

$$
H_{2}:=\left\{s>a: H^{1}\left(S \cap \partial B_{s}\right)>0\right\} .
$$

We have two cases to consider. Either $\left|H_{1}\right|=\infty$ or $\left|H_{2}\right|=\infty$. We will deal with each in turn. Firstly we will use a standard adaptation of Theorem $3.103[1]$ we have that for any $u \in L^{1}\left(B_{R}(0)\right)$

$$
V\left(u, B_{R}(0)\right)=\int_{0}^{R} V\left(u, \partial B_{s}(0)\right) \mathrm{d} s
$$

where

$$
V\left(u, \partial B_{s}(0)\right)=\sup \left\{\int_{0}^{2 \pi s} u\left(s \cos \left(\frac{\theta}{s}\right), s \sin \left(\frac{\theta}{s}\right)\right) \phi^{\prime}(\theta) \mathrm{d} \theta: \phi \in C_{0}^{\infty}([0,2 \pi s])\right\} .
$$

Now suppose $\left|H_{1}\right|=\infty$ then

$$
V\left(\mathbb{1}_{S}, \mathbb{R}^{2}\right) \stackrel{(6.50),(6.49)}{\geq} \int_{H_{1}} V\left(\mathbb{1}_{S}, \partial B_{s}(0)\right) \mathrm{d} s=\infty
$$

Which contradicts the fact that $S$ a set of finite perimeter. Now suppose $\left|H_{2}\right|=\infty$. Let $B_{2}:=$ $\left\{s \in H_{2}: H^{1}\left(\partial B_{s}(0) \cap S\right) \geq \pi s\right\}$ then by the Coarea formula

$$
\infty>|S| \geq \int_{s \in B_{2}} H^{1}\left(\partial B_{s}(0) \cap S\right) \mathrm{d} s \geq \int_{s \in B_{2}} \pi s \mathrm{~d} s \geq\left|B_{2} \cap\{x: x \geq 1\}\right| .
$$

Hence $\left|B_{2}\right|<\infty$. Now for any $t \in H_{2} \backslash B_{2}$ we must have $V\left(\mathbb{1}_{S}, \partial B_{s}(0)\right) \geq 1$. Thus as in (6.52), $V\left(\mathbb{1}_{S}, \mathbb{R}^{2}\right) \geq\left|H_{2} \backslash B_{2}\right|=\infty$ which contradicts the fact $S$ has finite perimeter. So in either case we have a contradiction, thus $b<\infty$.

Step 3. We will show $\left|{\overline{B_{b}(0)}}^{c} \cap S\right|=0$.

Proof of Step 3. Since $\left|D \mathbb{1}_{S}\right|\left({\overline{B_{b}(0)}}^{c}\right)=0$ either $\left|{\overline{B_{b}(0)}}^{c} \cap S\right|=0$ or $\left|{\overline{B_{b}(0)}}^{c} \cap S^{c}\right|=0$ in the latter case $S^{c}$ has an indecomposable component of finite measure which contradicts the fact that $S$ is stratified.

Step 4. We will show $S^{c}$ has only one indecomposable component. 
Proof of Step 4. Let $U_{1}, U_{2}, \ldots$ be the indecomposable components of $S^{c}$. Since $S$ is saturated, $\left|U_{k}\right|=\infty$ for any $k \in \mathbb{N}$. Thus $\left|U_{k} \cap B_{b}^{c}\right|>0$ for all $k$. Since $H^{1}\left(\partial^{M} U_{k} \cap B_{b}^{c}\right)=0$, thus $\left|D \mathbb{1}_{U_{k}}\right|\left(B_{b}^{c}\right)=0$ so either $\mathbb{1}_{U_{k}} \equiv 1$ on $B_{b}^{c}$ or $\mathbb{1}_{U_{k}} \equiv 0$ on $B_{b}^{c}$. As $\left|U_{k} \cap B_{b}^{c}\right|>0$ the former is true and so $\left|B_{b}^{c} \backslash U_{k}\right|=0$. Thus for every $k, l$ we have $\left|U_{k} \cap U_{l}\right|>0$ which is a contradiction. So there is only one indecomposable component.

Proof of Corollary completed. By Theorem 1.1, $S^{c}$ can be represented by an indecomposable component $\widetilde{\Pi}$ which is closed. So $\mathbb{S}:=(\widetilde{\Pi})^{c}$ then $\mathbb{S}$ is open and $D \mathbb{1}_{S}=D \mathbb{1}_{\mathbb{S}}$.

\section{Example: Proof of Theorem 1.3}

The construction. Let $\left\{\zeta_{k}: k \in \mathbb{N}\right\}$ be an enumeration of the points in the unit square in the $e_{1}, e_{2}$ plane with rational coordinates, i.e. points in the set $Q_{1}:=\left\{x e_{1}+y e_{2}: x, y \in[-1,1]\right\}$ that can be written as $x e_{1}+y e_{2}$ for some $x, y \in[-1,1] \cap \mathbb{Q}$.

We will define a thin column in direction $e_{3}$ around $\zeta_{k}$ by

$$
\Pi_{k}:=\left\{x e_{1}+y e_{2}+z e_{3}+\zeta_{k}: \sqrt{\left(x-\zeta_{k} \cdot e_{1}\right)^{2}+\left(y-\zeta_{k} \cdot e_{2}\right)^{2}}<2^{-10000-k}, 0<z \leq 1\right\} .
$$

And we define $\mathcal{O}=\bigcup_{k \in \mathbb{N}} \Pi_{k}$. Let

$$
\mathcal{U}=\{(x, y, z):-1 \leq x \leq 1,-1 \leq y \leq 1,-1 \leq z \leq 0\} .
$$

Finally let

$$
\mathcal{S}=\mathcal{O} \cup \mathcal{U}
$$

Since $\mathcal{O}$ and $\mathcal{U}$ are shaved so $\mathcal{U}$ is also a shaved set.

Step 1. We will show $\mathcal{S}$ is path connected.

Proof of Step 1. From the construction it is clear that for any $p \in \mathcal{S}$, the line interval $\left[p,\left(p \cdot e_{1}\right) e_{1}+\left(p \cdot e_{2}\right) e_{2}-\frac{e_{3}}{2}\right] \subset \mathcal{U}$. Thus for any $p_{1}, p_{2} \in \mathcal{O}$

$$
L_{1}=\left[p_{1},\left(p_{1} \cdot e_{1}\right) e_{1}+\left(p_{1} \cdot e_{2}\right) e_{2}-\frac{e_{3}}{2}\right] \subset \mathcal{S}
$$

and

$$
L_{2}=\left[p_{2},\left(p_{2} \cdot e_{1}\right) e_{1}+\left(p_{1} \cdot e_{2}\right) e_{2}-\frac{e_{3}}{2}\right] \subset \mathcal{S} .
$$

Finally

$$
L_{3}=\left[\left(p_{1} \cdot e_{1}\right) e_{1}+\left(p_{1} \cdot e_{2}\right) e_{2}-\frac{e_{3}}{2},\left(p_{2} \cdot e_{1}\right) e_{1}+\left(p_{1} \cdot e_{2}\right) e_{2}-\frac{e_{3}}{2}\right] \subset \mathcal{U} \subset \mathcal{S} .
$$

So

$$
L_{1} \cup L_{2} \cup L_{3} \subset \mathcal{S}
$$

Thus $p_{1}$ and $p_{2}$ are connected in $\mathcal{S}$.

Step 2. We will show $\mathcal{S}^{c}$ is connected.

Proof of Step 2. Let $Q=(-1,1) \times(-1,1) \times(-1,1)$. If $p \in Q \backslash \mathcal{S}$ then $p+\lambda e_{3} \notin \mathcal{S}$ for any $\lambda>0$. Thus given any two points $p_{1}, p_{2} \in Q \backslash \mathcal{S}$ we have $\left[p_{1}, p_{2}+2 e_{3}\right] \subset \mathcal{S}^{c}$ and $\left[p_{1}, p_{2}+2 e_{3}\right] \subset \mathcal{S}^{c}$ and thus the path

$$
\mathcal{P}=\left[p_{1}, p_{1}+2 e_{3}\right] \cup\left[p_{1}+2 e_{3}, p_{2}+2 e_{3}\right] \cup\left[p_{2}+2 e_{3}, p_{2}\right] \subset \mathcal{S}^{c},
$$

thus $\mathcal{P}$ is connected. 
Step 3. We will show $\mathcal{S}$ is a set of finite perimeter.

Proof of Step 3. Let $\mathcal{O}_{N}=\bigcup_{k=1}^{N} \Pi_{k}$ and define

$$
\mathcal{S}_{N}=\mathcal{O}_{N} \cup \mathcal{U}
$$

Note that $\mathcal{S}_{N}$ is a set of finite perimeter and

$$
\operatorname{Per}\left(\mathcal{S}_{N}\right) \leq \sum_{k=1}^{N} 2 \pi 2^{-10000-k}+17 \leq 18 .
$$

Now $\mathcal{S}_{N}$ converges in measure to $\mathcal{S}$ (see Rem. 3.36 [1]) so by Proposition 3.37 (b) we know $\operatorname{Per}(\mathcal{S}) \leq 18$ and thus $\mathcal{S}$ is a set of finite perimeter.

Step 4. For a.e. $p \in Q \backslash \mathcal{S}$ there exists $r_{p}>0$ such that

$$
\left|\mathcal{S} \cap B_{r}(p)\right|>0,\left|\mathcal{S}^{c} \cap B_{r}(p)\right|>0 \text { for all } r \in\left(0, r_{p}\right)
$$

and

$$
\operatorname{Per}\left(\mathcal{S}, B_{r}(p)\right)>0 \text { for any } r \in\left(0, r_{p}\right) .
$$

Proof of Step 4. By Lebesgue density theorem for a.e. $p \in Q \backslash \mathcal{S}$ there exists $r_{p}>0$ such that

$$
\frac{\left|B_{r}(p) \cap \mathcal{S}^{c}\right|}{\left|B_{r}(p)\right|}>\frac{1}{2} \text { for any } r \in\left(0, r_{p}\right] .
$$

Now for any $r \in\left(0, r_{p}\right]$ we can find $x, y \in \mathbb{Q}$ such that $\left(x, y, p \cdot e_{3}\right) \in \mathcal{O} \cap B_{\frac{r}{4}}(p)$ and as $\mathcal{O}$ is an open set for some $\rho \in\left(0, \frac{r}{4}\right)$ we know

$$
\left|B_{\rho}(p) \cap \mathcal{O}\right|>0 .
$$

So (7.7) and (7.6) together establish (7.4).

Now arguing by contradiction and assuming $\operatorname{Per}\left(\mathcal{S} \cap B_{r}(x)\right)=0$ then as this means $\left|D \mathbb{1}_{\mathcal{S}}\right|\left(B_{r}(x)\right)=0$ so by Theorem 3.44, [1] we have that $\int_{B_{r}(x)}\left|\mathbb{1}_{\mathcal{S}}-\left(\mathbb{1}_{\mathcal{S}}\right)_{B_{r}(x)}\right| \mathrm{d} z=0$. However by (7.7) and (7.6) and this is a contradiction. Thus (7.5) is established.

Step 5. We will show that $Q \backslash \mathcal{O} \subset \overline{\mathcal{S}}$.

Proof of Step 5. For any $p \in Q \backslash \mathcal{O}$ either we have $p \in \mathcal{U}$ or $p \in(Q \backslash \mathcal{U}) \backslash \mathcal{S}$. So using (7.4) of Step 4 for the latter case we know that for a.e. $p \in Q \backslash \mathcal{O}, p \in \overline{\mathcal{S}}$. So there exists a subset $\mathcal{G} \subset Q \backslash \mathcal{O}$ with $|(Q \backslash \mathcal{O}) \backslash \mathcal{G}|=0$ and $\mathcal{G} \subset \overline{\mathcal{S}}$. Thus $\overline{\mathcal{G}} \subset \overline{\mathcal{S}}$ and since $Q \backslash \mathcal{O} \subset \overline{\mathcal{G}}$ this completes the proof of Step 5 .

Proof of Theorem completed. By Step 1 and Step 2, $\mathcal{S}$ and $\mathcal{S}^{c}$ are connected. Since $\mathcal{S}$ is a set of finite perimeter by Proposition 2 [2] we have that $\mathcal{S}$ is an indecomposable set. Since $H^{2}\left(\partial^{M} \mathcal{S}^{c}\right)=H^{2}\left(\partial^{M} \mathcal{S}\right)<\infty$ in the same way by Proposition $2, \mathcal{S}^{c}$ is indecomposable. Thus it is its own only indecomposable component. Hence (1.3) is established.

Now note by Step $5, Q \backslash \mathcal{O} \subset \overline{\mathcal{S}}$ and

$$
|Q \backslash \mathcal{S}|=4-\sum_{k=1}^{\infty} \pi\left(2^{-10000-k}\right)^{2} \geq \frac{3999}{4000},
$$

so (1.3) is established. 
Finally by Step 4 we can find a set $\mathcal{G} \subset Q \backslash \mathcal{S}$ with $|(Q \backslash \mathcal{S}) \backslash \mathcal{G}|=0$ and for every $p \in \mathcal{G}$ there exists $r_{p}>0$ such that (7.5) holds true. So arguing by contradiction suppose there exists a Lipschitz map $\phi: S^{2} \rightarrow \mathbb{R}^{3}$ such that $H^{2}\left(\partial^{M} \mathcal{S} \backslash \phi\left(S^{2}\right)\right)=0$. Now by Theorems 3.59 and 3.61 [1],

$$
H^{2}\left(\phi\left(S^{2}\right) \cap B_{r}(p)\right) \geq H^{2}\left(\partial^{M} \mathcal{S} \cap B_{r}(p)\right)=\operatorname{Per}\left(\mathcal{S}, B_{r}(p)\right)>0 \text { for all } r \in\left(0, r_{p}\right) .
$$

So $p \in \phi\left(\bar{S}^{2}\right)=\phi\left(S^{2}\right)$. Thus $\mathcal{G} \subset \phi\left(S^{2}\right)$ but this is a contradiction because $H^{2}\left(\phi\left(S^{2}\right)\right) \leq(\operatorname{Lip}(\phi))^{2} H^{2}\left(S^{2}\right)=$ $4 \pi(\operatorname{Lip}(\phi))^{2}$. Thus we have established (1.3) and completed the proof of the theorem.

Remark 7.1. The example constructed in Theorem 1.3 also shows that Theorem 7 of [2] has no analogue in higher dimension. Note by property (1.3) there are no bounded components of $\mathcal{S}^{c}$ so $\mathcal{S}$ has no holes and is therefore saturated. Thus the set $\mathcal{S}$ constructed is an example of a set Ambrosio et al. define as simple (see Defs. 3 and $2[2])$. And by property (1.3) $\partial^{M} S$ can not be almost everywhere covered by the Lipschitz image of the sphere.

Acknowledgements. I would thank N. Shanmugalingam; the application to quasiminimizing sets was worked out through discussions with her. In addition I thank R.L. Jerrard who essentially provide me with the example constructed in Theorem 1.3. Finally I would like to thank the referee for careful reading and numerous excellent suggestions that greatly improved the readability of this note.

\section{REFERENCES}

[1] L. Ambrosio, N. Fusco and D. Pallara, Functions of bounded variation and free discontinuity problems. Oxford Mathematical Monographs. The Clarendon Press, Oxford University Press, New York (2000).

[2] L. Ambrosio, V. Caselles, S. Masnou and J.-M. Morel, Connected components of sets of finite perimeter and applications to image processing. J. Eur. Math. Soc. (JEMS) 3 (2001) 39-92.

[3] A. Baldi and F. Montefalcone, A note on the extension of BV functions in metric measure spaces. J. Math. Anal. Appl. 340 (2008) 197-208.

[4] Yu. Burago and V.G. Maz'ya, Potential theory and function theory for irregular regions. Translated from Russian. Seminars in Mathematics. Vol. 3. of V.A. Steklov Mathematical Institute, Leningrad, Consultants Bureau, New York (1969).

[5] G. David and S. Semmes, Quasiminimal surfaces of codimension 1 and John domains. Pacific J. Math. 183 (1998) $213-277$.

[6] J. Kinnunenm, R. Korte, A. Lorent and N. Shanmugalingam, Regularity of sets with quasiminimal boundary surfaces in metric spaces. J. Geom. Anal. 23 (2013) 1607-1640.

[7] B. Kirchheim, Lipschitz minimizers of the 3-well problem having gradients of bounded variation. MIS. MPg. Preprint (12/1998).

[8] P. Mattila, Geometry of sets and measures in Euclidean spaces. Fractals and rectifiability. Vol. 44. of Cambridge Studies in Advanced Mathematics. Cambridge University Press, Cambridge (1995). 